\title{
Second Generation Shock Tube Calibration System
}

\author{
David Wisniewiski \\ Meggitt Sensing Systems, San Juan Capistrano, CA 92675 USA
}

\begin{abstract}
(Received 31 October 2011; Provisionally Accepted 21 March 2012; Accepted 14 May 2012)
Meggitt Sensing Systems (formerly Endevco) pioneered the commercialization of a shock tube calibration system by taking an established laboratory tool capable of imparting near-instantaneous pressure stimulus and incorporating this capability into active product development for the purpose of providing quantifiable frequency response of pressure transducers. These activities commenced in 1996 in a cooperative effort with Texas Christian University, and results were reported extensively through 1998. After 15 years of successful new product introduction, empirical data has been used to model more accurately the 1-D compressible gas dynamics occurring within the shock tube so that the time interval of the reflected shock - the most critical parameter in determining the transfer function for the pressure transducer under test—can be optimized for varying amplitudes. The existing Meggitt Sensing Systems shock tube $(102 \mathrm{~mm}$ diameter $\times 3.7 \mathrm{~m}$ length) operates up to a pressure of $6895 \mathrm{kilopascal}$ gauge (kPag) and sustains up to $8 \mathrm{~ms}$ reflected shocks. Recent modelling suggests that these parameters can double. Accordingly, effort is currently under way to bring this enhanced capability to the marketplace, offering both increased performance and ease of user operation.
\end{abstract}

\section{NOMENCLATURE}

$\begin{array}{ll}P_{\text {shock }} & \text { fully developed shock wave pressure } \\ V_{\text {shock }} & \text { fully developed shock wave velocity } \\ V_{\text {sound_in_gas }} & \text { velocity of sound in gas } \\ P_{\text {ambient }} & \text { ambient pressure } \\ \Delta t & \text { maximum sustainable pressure step interval } \\ x_{i} & \text { 1-D position of compression wave, contact } \\ & \text { surface, and rarefaction wave } \\ x_{0 i} & \text { 1-D initial position of compression wave, } \\ & \text { contact surface, and rarefaction wave } \\ v_{0 i} & \text { 1-D initial velocity of compression wave, } \\ & \text { contact surface, and rarefaction wave } \\ a_{i} & \text { 1-D deceleration of compression wave, } \\ & \text { contact surface, and rarefaction wave }\end{array}$

\section{INTRODUCTION}

Shock tubes are a ubiquitous icon used in research activity across academic, government, and industrial settings. The most predominant example of those activities includes the study of gas reaction chemistry of a variety of substances (usually in the gas phase) since the shock tube can generate relatively high temperatures due to the compressive shock wave that is generated. A novel, recent application of the shock tube has been to focus on blast-induced traumatic brain injury on humans by subjecting laboratory animals to a shock impetus within a shock tube. ${ }^{1}$ This activity contrasts with larger shock tubes that actually blast sizable objects (from a meter tall to the size of a battle tank), treating them as artillery pieces.

Still, the most practical application of the shock tube, from an industrial perspective, is as a precision calibration device. ${ }^{2}$ In fact, the previous examples mentioned all require precise pressure instrumentation in order to accurately measure the pressure environment generated by the shock tube for the respective test. In effect, the shock tube is the vehicle for imparting near-perfect pressure stimulus for the purpose of calibrating the pressure instrumentation (i.e., the primary function), which subsequently supports all other offshoot testing applica-

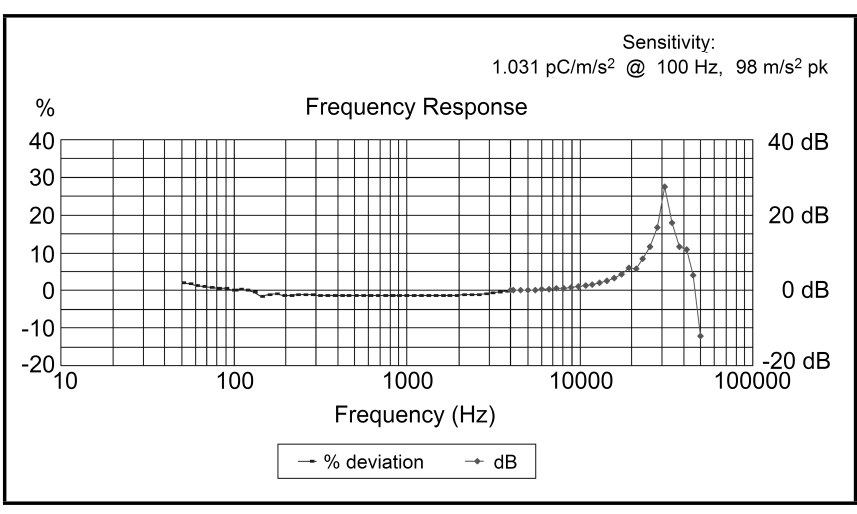

Figure 1. Accelerometer calibration certificate.

tions utilizing the calibrated pressure instrumentation (i.e., the secondary function).

This paper identifies the two most important performance parameters for dynamic pressure measurement while explaining their calibration significance from the context of another type of transducer (i.e., accelerometer). The shock tube is shown to be an excellent vehicle for obtaining these two most important performance parameters by presenting the methodology and actual data collected. Finally, a preview of the activity currently in progress on the Meggitt Sensing Systems second-generation shock tube is presented.

\section{ACCELEROMETER CONTEXT COMPARISON}

When procuring an accelerometer from any manufacturer, it is customary to receive a calibration certificate quantifying the dynamic performance parameters of the device. The two most important dynamic performance parameters for any accelerometer are sensitivity and frequency response. Moreover, it is expected that this information be provided with any accelerometer. Refer to Fig. 1 for a typical calibration certificate for an accelerometer. 